\title{
On the Dynamics and Stable Equilibria of Anti-symmetric CNNs
}

\author{
BaHram Mirzai, Drahoslav Lím and GeORge S. Moschytz \\ Signal and Information Processing Laboratory \\ Swiss Federal Institute of Technology \\ Zurich, Switzerland \\ mirzaieisi.ee.ethz.ch 1imaisi.ee.ethz.ch
}

\begin{abstract}
In this paper we investigate the dynamic behavior of the simplest anti-symmetric CNN. Stable equilibria of the system for constant boundary values are investigated. We provide a comparison with the simplest symmetric CNN in terms of dynamics and stable equilibria.
\end{abstract}

\section{Introduction}

In many modeling problems which utilize differential equations one is particularly interested in the stable equilibria of the underlying system. For example, patterns emerging in biological or chemical reactions may be considered to be the stable equilibria of such systems. In this work we consider the conventional CNN as the underlying modeling system and investigate its stable equilibria. We restrict our analysis to one dimensional CNN arrays, described by

$$
\frac{d x_{i}}{d t}=-x_{i}+\sum_{k \in \mathcal{N}_{i}} a_{i, k} f\left(x_{k}\right)+\sum_{k \in \mathcal{N}_{i}} b_{i, k} u_{k}+I
$$

for given initial conditions $x_{i}(0)$. The nonlinearity in (1) is the piecewise-linear function

$$
f(x)=\frac{1}{2}\{|x+1|-|x-1|\}
$$

and $\mathcal{N}_{i}$ is the set of neighbors connected to the $i$-th cell. The network parameters for space-invariant CNNs are given by a template set, consisting of the feedback template $A$, control template $B$ and the bias term $I$. The CNN inputs and outputs are defined to be $u_{i}$ and $f\left(x_{i}\right)$, respectively. The operation of a CNN consists of mapping an input image to its corresponding output at the equilibrium of the system. A classification of the possible outputs in terms of network parameters enables us to find the scope of patterns that can be associated with a template set.

In the following sections we investigate the dynamics and stable equilibria of the nearest neighbor anti-symmetric $\mathrm{CNN}$,

$$
\dot{x}_{i}=-x_{i}+s f\left(x_{i-1}\right)+p f\left(x_{i}\right)-s f\left(x_{i+1}\right),
$$

for fixed boundary values, and provide a comparison with the symmetric case,

$$
\dot{x}_{i}=-x_{i}+s f\left(x_{i-1}\right)+p f\left(x_{i}\right)+s f\left(x_{i+1}\right) .
$$

\section{Dynamic Behavior}

The dynamic behavior of (2) and (3) differs in a number of respects. In particular, the way in which some initial state evolves is quite different. As pointed out in [1], in the case of (3) the cells interact through a local diffusion process, whereas the interaction implied by (2) gives rise to wave-like propagating solutions. In many biological models, diffusion is used to describe locally and slowly interacting units (cells). In contrast, models admitting propagating behavior provide global and faster interaction among cells. This modeling aspect holds also for the above examples. The diffusion rate of (3) turns out to be smaller than the propagation velocity of (2) by an amount on the order of the grid size (spatial quantization step). Hence, in the antisymmetric case, the range of interaction and information transmission is considerably higher than in the symmetric case.

To elaborate on these ideas in some detail, let us derive in each case the non-linear PDE that gives rise to (2) and (3), respectively. It is easily shown that the discretization of

$$
\begin{aligned}
\partial_{t} u(x, t)= & -u(x, t)+(p+2 s) f(u(x, t)) \\
& +D \partial_{x}^{2} f(u(x, t))
\end{aligned}
$$

according to $u(x, t) \rightarrow x_{i}(t)$ and

$$
\partial_{x} u(x, t) \rightarrow d_{F} x_{i}(t):=\frac{x_{i+1}(t)-x_{i}(t)}{h},
$$

leads to (3) if $D=s h^{2}$, where $d_{F}$ is the forward approximation to $\partial_{x}$. Similarly, discretizing

$$
\begin{aligned}
\partial_{t} u(x, t) & +c \partial_{x} f(u(x, t))= \\
& -u(x, t)+p f(u(x, t)),
\end{aligned}
$$

by means of the centered approximation of the derivative,

$$
\partial_{x} u(x, t) \rightarrow d_{C} x_{i}(t):=\frac{x_{i+1}(t)-x_{i-1}(t)}{2 h},
$$

we obtain (2) if $c=2 s h$.

In the linear region of $f(\cdot)$, equation (4) corresponds to a damped or amplified diffusion, depending on the sign of $p+2 s-1$ :

$$
\partial_{t} u(x, t)=-u(x, t)+(p+2 s) u(x, t)+D \partial_{x}^{2} u(x, t) .
$$


This follows from the fact that by the substitution

$$
u(x, t)=e^{(p+2 s-1) t} v(x, t),
$$

$v(x, t)$ satisfies the well known heat equation.

In [2] a rigorous classification of the stable equilibria of (3) was given. Here we provide a heuristic explanation of those results from the view point of (4). The analysis can be done in terms of the parameter $\mu=(p-1) / 2 s$. In the simplest case, $\mu=-1$, equation (4) corresponds to the heat equation with solutions converging to the boundary value. For $\mu<-1$ and zero or periodic boundary values, the origin is the only (asymptotically) stable equilibrium as suggested by (6). For $\mu>1$, the diffusion process in (4) is dominated by the contribution of self-feedback. To see this, note that $2 s$ in $e^{(p+2 s-1) t}$ corresponds to the diffusion term $D \partial_{x}^{2}$ in (4). Hence, the condition $\mu>1$, i.e., $p-1>2 s$, implies that the contribution due to the self-feedback exceeds that of the diffusion in the exponential. In the discrete case this corresponds to the fact that any sequence of bipolar inputs is a stable equilibrium. Finally, for $-1<\mu<1$, the diffusion and the self-feedback are two competing processes and the resulting equilibria are classified in [2].

Let us now consider (5) in the linear region of $f(\cdot)$,

$$
\partial_{t} u(x, t)+c \partial_{x} u(x, t)=-u(x, t)+p u(x, t) .
$$

By the substitution $u(x, t)=e^{(p-1) t} v(x, t)$ and the coordinate transformation $x^{\prime}=x-c t, t^{\prime}=t$, we obtain from (7)

$$
\partial_{t^{\prime}} v\left(x^{\prime}, t^{\prime}\right)=0 \text {. }
$$

Solutions of (7) in terms of $v(x, t)$ are then given by

$$
u(x, t)=e^{(p-1) t} v(x-c t, t) .
$$

The $x-c t$ dependence of the solution (9) implies its propagating nature and a propagation velocity $c$.

Under time reversal, $t \rightarrow-t$, equation (7) becomes

$$
\partial_{t} u(x, t)-c \partial_{x} u(x, t)=+u(x, t)-p u(x, t),
$$

the solutions of which have the form $u(x, t)=$ $e^{-(p \cdot l) t} v(x+c t, t)$, with $v(x, t)$ being a solution of (8) as well. Hence (5) can have both leftward and rightward propagating solutions. This symmetry of the continuous case is reflected in the discrete case by the fact that reversing the sign of $s$ in the $A$ template, i.e., $A=[-s p s]$, changes the propagation direction $(c \rightarrow-c)$.

Comparing now the diffusion coefficient $D=s h^{2}$ with the propagation velocity $c=2 h s$, it follows that the rate of interaction in the symmetric case is slower by a factor of $h$ than the anti-symmetric case.

Note that by discretizing (4) and (5) we assumed different approximations of the derivative $\partial_{x}$. In fact, the centered approximation has an error on the order of $O\left(h^{2}\right)$ smaller than the forward approximation, where the error is of the order of $O(h)$. Clearly (4) cannot be derived as the discrete version of a PDE by using $d_{C}$, and an attempt to derive (5) from a PDE by means of $d_{F}$ would imply a higher approximation error.

\section{Stable Equilibria}

In this section we investigate the stable equilibria of the system (5) for constant boundary values, and in particular for the values \pm 1 . In some cases, as discussed below, this turns out not to be a severe restriction. A classification of the equilibria based on approximative methods for the periodic boundary values is given in [3]. In [4] conditions for existence of stable equilibria in the case of $p>1$ and zero boundary conditions are considered.

Let $N$ be the length of the CNN array, excluding the boundary cells, and $\mu=(p-1) / 2 s$. Further, denote the boundary values by $x_{0}^{*}$ and $x_{N+1}^{*}$. We distinguish the following cases in terms of $\mu$ :

Case 1: $\mu<0$.

Consider the stable equilibria of the linear system,

$$
\dot{\mathbf{x}}=-\mathbf{x}+\mathbf{A} \mathbf{x}+\mathbf{b},
$$

where

$$
\mathbf{A}=\left(\begin{array}{ccccc}
p & -s & 0 & & 0 \\
s & p & -s & \ddots & \\
0 & \ddots & \ddots & \ddots & 0 \\
& \ddots & s & p & -s \\
0 & & 0 & s & p
\end{array}\right), \mathbf{b}=\left(\begin{array}{c}
s x_{0}^{*} \\
0 \\
\vdots \\
0 \\
-s x_{N+1}^{*}
\end{array}\right)
$$

and $\mathbf{b}$ contains the contribution of the constant boundary cells. The solution of $(11)$ for some initial value $\mathbf{x}(0)$ is

$$
\mathbf{x}(t)=e^{(\mathbf{A}-1) t} \mathbf{x}(0)+\left(e^{(\mathbf{A}-1) t}-\mathbf{1}\right) \mathbf{A}^{-1} \mathbf{b},
$$

with 1 being the identity matrix. Clearly, the stability of the solutions depends on the eigenvalues of $\mathbf{A}-\mathbf{1}$. To derive stability conditions, we decompose $\mathbf{A}$ into $\mathbf{A}=p \mathbf{1}+\mathbf{B}$, where $\mathbf{B}$ is the anti-symmetric part of $\mathbf{A}$. Due to the commutation of $(p-1) \mathbf{1}$ with $\mathbf{B}$, i.e., $[(p-1) \mathbf{1}, \mathbf{B}]=\mathbf{0}^{\dagger}$, we obtain $e^{(\mathbf{A}-1) t}=e^{(p-1) t} e^{\mathbf{B} t}$. Since the eigenvalues of $\mathbf{B}$ are imaginary, the stability of the equilibrium is guaranteed as long as $p-1<0$. Note that in contrast to the symmetric case, the stability condition does not impose any constraints on $\mu$ in terms of the array size $N$.

The following theorem can be proved for the equilibria of the linear system (11). For a partial proof see the Appendix; due to limited space, the full proof will be presented elsewhere [5].

Theorem: For constant boundary values the stable equilibria of the system (11) are characterized as follows. Let

$$
x_{l}^{*}=\frac{1}{\Delta_{N}}\left(s^{N-l+1} \Delta_{l-1} x_{N+1}^{*}+(-1)^{l} s^{l} \Delta_{N-l} x_{0}^{*}\right),
$$

with

$$
\Delta_{n}= \begin{cases}\frac{\cosh ((n+1) \alpha)}{\cosh (\alpha)} s^{n} & \text { if } n \text { even } \\ \frac{\sinh ((n+1) \alpha)}{\cosh (\alpha)} s^{n} & \text { if } n \text { odd }\end{cases}
$$

where $\sinh (\alpha)=\mu$ and $\Delta_{0}=1$.

${ }^{+}$The commutator $[M, N]$ of two matrices $M$ and $N$ is defined by $[M, N]=M N-N M$ 
I) If $x_{0}^{*}=x_{N+1}^{*}= \pm 1$

\section{For $N$ even, define $\alpha_{e}$ to be the solution of}

$$
\cosh \left((N+1) \alpha_{e}\right)+\sinh \left(N \alpha_{e}\right)-\cosh \left(\alpha_{e}\right)=0 .
$$

If $\alpha<\alpha_{e}$, then the equilibrium is given by (13). How ever, if $\alpha>\alpha_{e}$, then $f\left(x_{1}^{*}\right)=\operatorname{sign}\left(x_{0}^{*}\right)$ and the remaining cells assume the equilibrium values given by (13) for an array of length $N-1$ and the same $\alpha$ and boundary values.

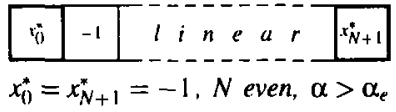

2. For $N$ odd, the stable equilibrium is given by (13) for all $\alpha<0$.

II) If $x_{0}^{*}=-x_{N+1}^{*}= \pm 1$

1. For $N$ even, and if $\alpha<\alpha_{e}, \alpha_{e}$ defined as above, the equilibrium is given by (13). However, if $\alpha>\alpha_{e}$, then $f\left(x_{N}^{*}\right)=\operatorname{sign}\left(x_{0}^{*}\right)$ and the remaining $N-1$ cells assume their equilibrium according to (13), with the same $\alpha$ and boundary values given by $\operatorname{sign}\left(x_{0}^{*}\right)$.

2. For $N$ odd, let $\alpha_{0}^{\ddagger}$ be the solution of

$$
\sinh \left((N+1) \alpha_{o}\right)+\cosh \left(N \alpha_{o}\right)+\cosh \left(\alpha_{o}\right)=0 .
$$

If $\alpha<\alpha_{0}$, then the stable equilibrium is given by (13). If $\alpha>\alpha_{0}$, then $f\left(x_{1}^{*}\right)=f\left(x_{N}^{*}\right)=\operatorname{sign}\left(x_{0}^{*}\right)$ and the remaining cells take on the equilibrium of an array of $N-2$ cells surrounded by two saturated cells of the color $\operatorname{sign}\left(x_{0}^{*}\right)$.

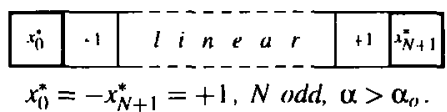

Simulations were performed on arrays of length $N=$ 15 and 16 , and with $x_{0}^{*}=x_{N+1}^{*}=-1$ and $\alpha>\alpha_{e}$. The first plot in Fig. 1 corresponds to the trajectory of $x_{1}^{(16)}$ of an $N=16$ array. The second plot depicts the remaining trajectories, i.e., $x_{2}^{(16)}, \ldots, x_{16}^{(16)}$, and the third plot shows the trajectories of an $N=15$ array. Observe that at the equilibrium point $f\left(x_{1}^{(16)}\right)=-1$ and $x_{l}^{(15)}=x_{l+1}^{(16)}$, as claimed by the theorem.

The generalization of the theorem to the case $\left|x_{0}^{*}\right|=$ $\left|x_{N+1}^{*}\right| \in \mathbb{R}_{+}$is obtained by normalizing the states to $x_{1} /\left|x_{0}^{*}\right|$. This reduces the analysis to the previous theorem. The case $\left|x_{0}^{*}\right| \neq\left|x_{N+1}^{*}\right| \in \mathbb{R}_{+}$is along the same lines.

Case 2: $0<\mu<1$.

In this region of $\mu$, for $x_{0}^{*}=x_{N+1}^{*}= \pm 1$, the CNN operates as a connected component detector $(\mathrm{CCD})^{\S}$. Hence, assuming a bipolar initial state, the corresponding stable

\footnotetext{
${ }^{\ddagger}$ The subscript stands for odd.

setting the boundary to +1 inverts the final output.
}

equilibrium is going to be the output of the $\mathrm{CCD}$ initialized with this particular initial configuration. For example, with $x_{0}^{*}=x_{N+1}^{*}=-1$ and $N=5$, only the following stable equilibria exist:

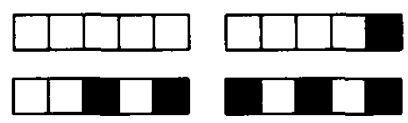

The generalization to arbitrary $N$ is obvious. A proof of this result is given in [6].

Case 3: $\mu>1$.

For $\left|x_{0}^{*}\right| \geq 1$ and $\left|x_{N+1}^{*}\right| \geq 1$ any bipolar sequence of cells is an equilibrium of the system (11). This easily follows from the estimate

$$
\left|x_{l}^{*}\right|=\left|s f\left(x_{l-1}^{*}\right)+p f\left(x_{l}^{*}\right)-s f\left(x_{l+1}^{*}\right)\right| \geq p-2 s>1 .
$$

If the magnitude of the boundary values is less than one, then in order for a bipolar sequence to still be a stable equilibrium of the system (11), the bound for $\mu$ must be modified to $\mu>(1+\xi) / 2$, where $\xi=\min \left\{\left|x_{0}^{*}\right|,\left|x_{N+1}^{*}\right|\right\}$. Assuming $\xi=\left|x_{0}^{*}\right|$, we then obtain

$$
\left|x_{1}^{*}\right|=\left|s f\left(x_{0}^{*}\right)+p f\left(x_{1}^{*}\right)-s f\left(x_{2}^{*}\right)\right| \geq p-(1+\xi) s>1 .
$$

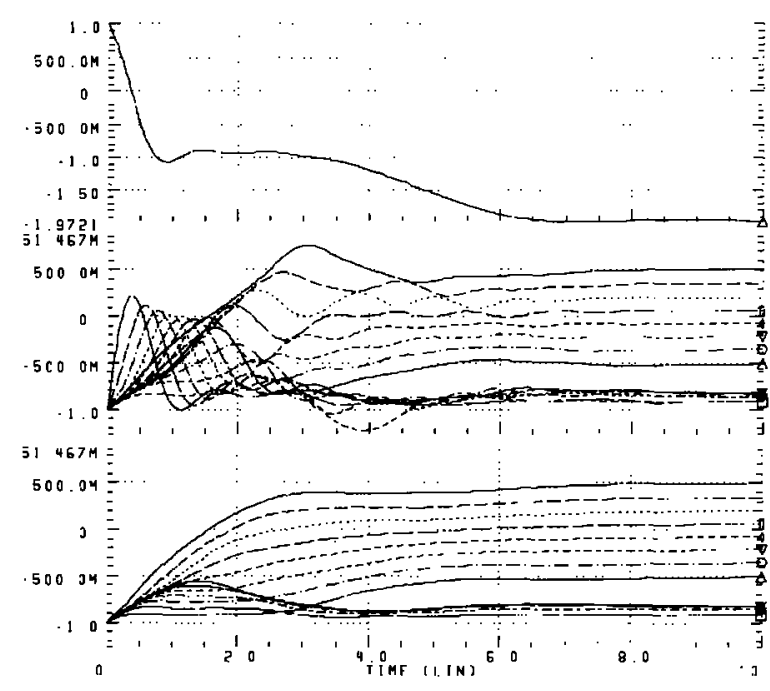

Figure 1: State trajectories

\section{Conclusions}

We considered the dynamic behavior and stable equilibria of the simplest anti-symmetric CNN with fixed boundary values. Furthermore, we provided a comparison with the symmetric case. In terms of the dynamics, the symmetric template corresponds to a diffusion process, whereas the anti-symmetric template corresponds to a propagation process. The diffusion constant turns out to be smaller than 
the convection (propagation) velocity by a factor on the order of the grid spacing. The stable equilibria of the anti-symmetric template can be divided into three classes. In each case we provided a classification of the equilibria for different boundary values. Note that in the anti-symmetric case it is possible to assign to each initial state its corresponding output.

\section{Appendix}

Proof of Theorem. From (11) it follows that the equilibrium is the solution of

$$
\mathbf{x}^{*}=\mathbf{A} \mathbf{x}^{\mathbf{x}}+\mathbf{b},
$$

i.e., $\mathbf{x}^{*}=-(\mathbf{A}-\mathbf{1})^{-1} \mathbf{b}$. Define $\mathbf{M}=\mathbf{A}-\mathbf{1}$. The equilibrium can be written as

$$
x_{i}^{*}=\frac{s}{\operatorname{det}(\mathbf{M})}\left(\operatorname{adj}(\mathbf{M})_{l N} x_{N+1}^{*}-\operatorname{adj}(\mathbf{M})_{l 1} x_{0}^{*}\right) .
$$

where $\operatorname{adj}(\mathbf{M})_{i j}=(-1)^{i+j} \operatorname{det}(\mathbf{M}(j \mid i))$ and $\mathbf{M}(j \mid i)$ is the matrix obtained by removing the $j$-th row and $i$-th column of $\mathbf{M}$. The following two equations can be shown to hold:

$$
\begin{aligned}
\operatorname{det}(\mathbf{M}(1 \mid l)) & =s^{l-1} \Delta_{N-l} \\
\operatorname{det}(\mathbf{M}(N \mid N-l+1)) & =(-1)^{l-1} s^{l-1} \Delta_{N-l},
\end{aligned}
$$

where $\Delta_{n}$ is the determinant of $\mathbf{M}_{n}$, the $n \times n$-dimensional version of $\mathbf{M}$, and $\Delta_{0}=1$. Hence $\operatorname{adj}(\mathbf{M})_{l \mathbf{l}}=$ $(-1)^{l-1} s^{l-1} \Delta_{N-l}$, and $\operatorname{adj}(\mathbf{M})_{l N}=s^{N-l} \Delta_{l-1}$, respectively. Inserting these into (15) implies (13).

To determine $\Delta_{h}$, the following recursion can be derived:

$$
\Delta_{n}=(p-1) \Delta_{n-1}+s^{2} \Delta_{n-2} .
$$

By the change of variable $s=i t, i=\sqrt{-1}$, we obtain

$$
\Delta_{n}=(p-1) \Delta_{n-1}-t^{2} \Delta_{n-2},
$$

with $t \in \mathbb{C} \backslash\{0\}$. By $\Delta_{n}=t^{n} U_{n}$, it follows from (16)

$$
U_{n}=2 \frac{p-1}{2 t} U_{n-1}-U_{n-2} \text {. }
$$

Solutions of (17) are Chebyshev polynomials of the second kind given by

$$
U_{n}(z)=\frac{\left(z+\sqrt{z^{2}-1}\right)^{n+1}-\left(z-\sqrt{z^{2}-1}\right)^{n+1}}{2 \sqrt{z^{2}-1}},
$$

where $z=\frac{p-1}{2 t}=-i \mu$. After some calculation, (18) can be written as

$$
U_{n}(\alpha)= \begin{cases}-(-i)^{n+2} \frac{\cosh ((n+1) \alpha)}{\cosh (\alpha)} s^{n} & \text { if } n \text { even, } \\ (-i)^{n+2} \frac{\sinh ((n+1) \alpha)}{\cosh (\alpha)} s^{n} & \text { if } n \text { odd }\end{cases}
$$

where $\mu=\sinh (\alpha)$. Equation (14) then follows.

We continue by showing that for $N$ even and $x_{0}^{*}=x_{N+1}^{*}= \pm 1$ there exists $\alpha_{e}$ below which the equilibria are given by (13). For $l$ odd, we obtain from (13-14)

$$
\begin{aligned}
\left|x_{l}^{*}\right| & =\frac{\left|\cosh (l \alpha) x_{N+1}^{*}-\sinh ((N-l+1) \alpha) x_{0}^{*}\right|}{\cosh ((N+1)|\alpha|)} \\
& =\frac{\cosh (l \alpha)+\sinh ((N-l+1)|\alpha|)}{\cosh ((N+1) \alpha)} \leq\left|x_{1}^{*}\right|
\end{aligned}
$$

Similarly, for $l$ even, it can be shown that $\left|x_{l}^{*}\right| \leq\left|x_{1}^{*}\right|$. For $x_{1}$ to have its equilibrium in the linear region, we require

$$
\left|x_{1}^{*}\right|=\frac{\cosh (\alpha)+\sinh (N|\alpha|)}{\cosh ((N+1) \alpha)} \leq 1
$$

which implies the corresponding equation for $\alpha_{e}$ in the theorem. Hence, if $\alpha<\alpha_{e}$ the equilibrium is given by (13). Otherwise, if $\alpha>\alpha_{e}$ the cell $x_{1}$ saturates with the same output color as $x_{0}^{*}$ and the remaining cells correspond to an array of length $N-1$ (odd), surrounded by two cells of the same color. This configuration admits an equilibrium according to (13) as shown below.

We now consider the case $N$ odd and $x_{0}^{*}=x_{N+1}^{*}= \pm 1$, and show that for all $\alpha$ the equilibrium is given by (13). We consider here only the case of even $l$. From (13-14) it follows that

$$
\begin{aligned}
\left|x_{l}^{*}\right| & =\frac{\left|\sinh (l \alpha) x_{N+1}^{*}+\sinh ((N-l+1) \alpha) x_{0}^{*}\right|}{|\sinh ((N+1) \alpha)|} \\
& =\frac{\sinh (l|\alpha|)+\sinh ((N-l+1)|\alpha|)}{\sinh ((N+1)|\alpha|)} .
\end{aligned}
$$

This and the inequality

$$
\sinh (x)+\sinh (y) \leq \sinh (x+y) \forall x, y>0
$$

imply that $\left|x_{l}^{*}\right| \leq 1$. Similarly, this can be shown for odd $l$.

So far, we have only considered the case $x_{0}^{*}=x_{N+1}^{*}=$ \pm 1 . The proofs for the case $x_{0}^{*}=-x_{N+1}^{*}= \pm 1$ are omitted due to space limitation, and can be found in [5].

\section{References}

[1] P. Thiran, G. Setti, and K. R. Crounse, "Spatio-Temporal Patterns in Cellular Neural Networks," in IEEE International Symposium On Circuits And Systems, vol. 3, (Atlanta), pp. 142-145, May 1996.

[2] P. Thiran, K. R. Crounse, L. O. Chua, and M. Hasler, "Pattern Formation Properties of Autonomous Cellular Neural Networks," IEEE Transactions on Circuits and Systems-I, vol. 42, pp. 757-774, Oct. 1995.

[3] K. R. Crounse, P. Thiran, G. Setti, and L. O. Chua, "Characterization and Dynamics of Pattern Formation in Cellular Neural Networks," to be published.

[4] S. Arik and V. Tavsanoglu, "Equilibrium Analysis of Non-symmetric CNNs," International Journal of Circuit Theory and Applications, vol. 24, pp. 275-281, May 1996.

[5] B. Mirzai, D. Lím, and G. S. Moschytz, "On Stable Equilibria of Opposite Sign Templates," 1996. in preparation.

[6] B. Mirzai, D. Lím, and G. S. Moschytz, "Robust Template Design Methods for CNNs," Sept. 1996. submitted for publication. 